\title{
Sobre Darwin y su influencia en la ciencia: una interpretación pragmatista de la estadística inglesa de fines del siglo XIX
}

\author{
Juan Ignacio Piovani \\ jpiovani@unibo.edu.ar
}

\section{Resumen}

En este artículo se examina la recepción que la obra de Darwin tuvo hacia fines de siglo XIX y principios del siglo XX en el pensamiento anglosajón, en particular en el marco de corrientes positivistas y pragmatistas. Se presta especial atención a algunas de las figuras más empeñadas en la defensa de la ciencia, tales como Galton, Pearson y Dewey, quienes creían que On the Origin of Species había introducido un nuevo modo de pensar, transformando la lógica del conocimiento. En sentido estricto, en este trabajo interesa analizar los aportes de Galton y Pearson a la teoría estadística a partir del esquema con el que Dewey interpretó a la obra de Darwin y sus consecuencias para las ciencias y para el pensamiento contemporáneo.

Palabras clave: ciencia, positivismo, pragmatismo, estadística.

\begin{abstract}
This essay examines the reception that Darwin's work had by the end of the 19th century and the beginning of the 20th century in the Anglo-Saxon thought, particularly among positivist and pragmatist perspectives. Special attention is given to some of the authors most engaged in the defence of science, such as Galton, Pearson and Dewey, who maintained that Darwin's On the Origin of Species had introduced a new way of thinking, transforming the logic of knowledge. More specifically, the main objective of this article is to analyse Galton and Pearson's contributions to statistical theory from the standpoint of Dewey's interpretation of the consequences that the work by Darwin had had for science and contemporary thought.
\end{abstract}

Keywords: science, positivism, pragmatism, statistics.

*Juan Ignacio Piovani es Doctor de Metodología de las Ciencias Sociales por la Università di Roma "La Sapienza" y Profesor Titular Ordinario en la Facultad de Humanidades de la Universidad Nacional de La Plata. 
La era victoriana ha sido frecuentemente caracterizada como la "edad de la ciencia”. Sin embargo, ésta no había alcanzado entonces un completo éxito en sus reivindicaciones: su rol social, cultural e intelectual era objeto de encendidos debates. ${ }^{1}$ En las primeras décadas del siglo XIX muchos pensadores ingleses intentaban monopolizar el modo "correcto" de definir a la ciencia. No se había consolidado aún una comunidad científica que pudiese arrogarse legítimamente la autoridad sobre el conjunto de reglas que "deberían" definir la práctica científica. ${ }^{2}$

En una reconstrucción ex post, resulta relativamente fácil encontrar en el pensamiento y los descubrimientos científicos que marcan el inicio de la modernidad, la semilla que habría terminado por destruir todo rastro de la visión teológicometafísica de la realidad. Pero en su infancia, la emergente ciencia moderna debió adaptarse a esta concepción, que era todavía dominante. Fue recién en el siglo XIX que la tensión entre estas dos cosmologías - la teológico-metafísica y la científiconaturalista - estaba alcanzando un punto culminante.

Fayter $^{3}$ afirma que esta crisis comenzó en 1798 con la publicación de Essays on the Principles of Population, de Thomas Malthus, y llegó al clímax con la obra de Darwin On The Origin of Species (1859), la cual situaba al hombre en un contexto cósmico que negaba la historia bíblica de la creación. Según Lightman ${ }^{4}$, la obra de Darwin produjo la fractura del contexto intelectual común asegurado por la teología natural. De un lado, los intelectuales conservadores comenzaron a sentirse cada vez más bajo la amenaza del pensamiento naturalista y materialista. Del otro lado, los hombres de ciencia temían que su proyecto científico y la difusión de su ideología

\footnotetext{
${ }^{1}$ Véase Kjaergaard, P. C., Defending Science: 'Genuine Scientific Men' and the Limits of Natural Knowledge. Aarhus, University Press, 2000.

${ }^{2}$ Winter, A., "The Construction of Orthodoxies and Heterodoxies in the Early Victorian Life Sciences", en Lightman, B. (ed.), Victorian Science in Context, Chicago, University Press, 1997, pp. 24-50.

${ }^{3}$ Fayter, P., "Strange New Worlds of Space and Time: Late Victorian Science and Science Fiction", en Lightman, Bernard (ed.), op. cit., pp. 256-280.

${ }^{4}$ Lightman, B., "'The Voices of Nature': Popularizing Victorian Science", en Lightman, B. (ed.), op. cit., pp. 187-211.
} 
fracasasen, y en consecuencia reivindicaban — justamente porque sus pretensiones eran desafiadas - el derecho exclusivo para definir el conocimiento natural. ${ }^{5}$

El conflicto entre el naturalismo y las visiones tradicionales del mundo típico de los períodos medio y tardo victorianos - no fue por lo tanto una mera confrontación de ideas abstractas; en discusión estaba la autoridad de pronunciarse sobre el cosmos, la sociedad y el ser humano - y obviamente la capacidad de apropiarse de las "ventajas terrestres" que se derivan de tal autoridad.

En el marco de la tensión apenas señalada, este artículo se aboca al examen de la recepción que la obra de Darwin tuvo hacia fines de siglo XIX y principios del siglo XX en el pensamiento anglosajón, en particular en algunas corrientes positivistas y pragmatistas. En este sentido, se dedica especial atención a algunas de las figuras más empeñadas en la defensa de la ciencia, que como Francis Galton, Karl Pearson y John Dewey, creían que On the Origin of Species había introducido un nuevo modo de pensar, transformando la lógica del conocimiento. ${ }^{6}$ Más específicamente, interesa analizar las contribuciones de Galton y Pearson a la teoría estadística $^{7}$ a la luz del esquema interpretativo con el que Dewey caracterizó a la obra de Darwin y sus consecuencias para las ciencias y el pensamiento contemporáneo.

Para Dewey, Darwin no fue el primero en cuestionar la filosofía clásica de la naturaleza y del conocimiento, pero su obra constituye el último hito científico en esa dirección:

Sin los métodos de Copérnico, Kepler, Galileo y sus sucesores en la astronomía, la física y la química, Darwin se habría encontrado desasistido en las ciencias orgánicas. No obstante, antes de Darwin, el impacto del nuevo método científico sobre la vida, la mente

\footnotetext{
${ }^{5}$ Véase Kjaergaard, op. cit.

${ }^{6}$ Véase por ejemplo Galton, F., Natural inheritance, 1889; Pearson, K., The Grammar of Science, Londres, Scout, 1892 [citas de la tercera revisión (1911), Nueva York, Meridian, 1957]; Dewey, J., "The influence of Darwinism on Philosophy", en The influence of Darwinism on Philosophy and other essays in contemporary thought, Nueva York, Henry Holt and Co., 1910 [citas de la trad. esp.: "La influencia del darwinismo en la filosofía", en La miseria de la epistemología. Ensayos de pragmatismo, Madrid, Biblioteca Nueva, 2000, pp. 49-60].

${ }^{7}$ Galton y Pearson son considerados figuras fundamentes de la moderna teoría estadística. Por teoría estadística entiendo "un marco teorético para el análisis de datos numéricos" y un conjunto de "instrumentos que pueden ser usados" a tal fin (MacKenzie, D., Statistics in Britain, 1865-1930. The Social Construction of Scientific Knowledge, Edimburgo, University Press, 1981, p. 7).
} 
y la política no podía producirse, pues entre esos intereses ideales o morales y el mundo inorgánico se interponía el reino de plantas y animales. Por lo tanto, él fue quien hizo posible que 'la nueva lógica se aplicase a la mente, a la moral y a la vida'. ${ }^{8}$

Galton y Pearson, reconociéndose herederos de Darwin, fueron justamente dos de los científicos más notables entre aquellos que se dedicaron a desarrollar y aplicar esta nueva lógica en los "reinos de plantas y animales", y de extenderla progresivamente a los fenómenos mentales, sociales y políticos. Su contribución fundamental se encuentra en las herramientas técnicas de análisis estadístico que idearon a tal fin —especialmente la correlación ${ }^{9}$ - , y que desde su perspectiva serían capaces de elevar al rango de "verdaderas ciencias" a todas las disciplinas que se ocupaban de los fenómenos apenas mencionados. En palabras del propio Pearson, pronunciadas en los últimos años de su vida, al recordar la "promesa" que para él y sus colaboradores habían conllevado estos nuevos instrumentos científicos en los momentos en que les estaban dando forma:

\footnotetext{
La correlación llevaba en gran medida a la psicología, la antropología, la medicina y la sociología dentro del campo del tratamiento matematico [...] Se daba así por primera vez una posibilidad, no quiero decir la certeza, de alcanzar conocimeintos tan válidos como se consideraba entonces a los conocimientos físicos - en el campo de las formas vivientes, y sobretodo, en el campo del comportamiento humano. ${ }^{10}$
}

A los ojos de estos dos grandes estadísticos, tal como la cita precedente sugiere, la correlación representaba la máxima realización de la nueva perspectiva científica: un instrumento que permitiría investigar empíricamente las cuestiones orgánicas, morales y sociales, entre otras, estableciendo relaciones (expresables

\footnotetext{
${ }^{8}$ Dewey, J., op. cit., pp. 53-54.

${ }^{9}$ La correlación, en sentido estadístico, se refiere en términos genéricos a la magnitud y dirección de una relación. En la ciencia esto se expresa habitualmente de modo cuantitativo a través de coeficientes, como el $r$ de Parson (Pagano, R., Understanding statistics in the behavioral sciences, Pacific Grove, Brooks/Cole, 1998).

10 Discurso leído en el University College de Londres, en un acto en honor al Prof. Karl Pearson, 23 de abril de 1934. Citado por Pearson, E., Karl Pearson: An Appreciation of Some Aspects of his Life and Work, Cambridge, University Press, 1938. [citas de la trad. esp.: Pearson, creador de la estadística aplicada, Buenos Aires, Espasa-Calpe, 1948, p. 46].
} 
cuantitativamente) entre fenómenos observados. De este modo podría cumplirse al fin, también en el ámbito de las disciplinas no experimentales, el supuesto galileano — central en la visión canónica de la ciencia - según el cual el objetivo principal de la empresa científica consiste en el control empírico de aserciones (hipotéticas) que expresan relaciones entre propiedades de los objetos ${ }^{11}$, y cuyo logro en la física y la química lo garantizaba instrumentalmente la experimentación. Por otra parte, estas relaciones empíricas no se definían en los clásicos términos metafísicos de "causas y efectos" necesarios:

\begin{abstract}
Que el universo sea una suma de fenómenos, algunos más y otros menos ligados entre sí, es la nueva concepción [...] que al presente debemos extraer de nuestra experiencia [...] El objetivo de la ciencia [...] consiste en buscar los fenómenos más fuertemente correlacionados [...] Ningún fenómeno es causal; todos son contigentes, y el problema que debemos afrontar es el de medir su grado de dependencia. ${ }^{12}$
\end{abstract}

Tanto para Galton y Pearson, como para Dewey, era justamente la idea metafísica de causa - a su juicio totalmente injustificada - lo que la obra de Darwin había ayudado a desenmascarar definitivamente en toda su irracionalidad. Esta crítica de la "metafísica de la causalidad", que muy especialmente Pearson trató de reemplazar con una "ciencia de correlaciones", constituye uno de los puntos en común más notables de la interpretación que estos autores, a ambos lados del Atlántico y en el marco de perspectivas epistemológicas diferentes, hicieron de las consecuencias del evolucionismo darwinista.

Para Dewey, la idea de causalidad formaba parte de una concepción reinante en Occidente por más de 2000 años; un principio del conocimiento y de la naturaleza sobre el que se había hecho descansar la lógica de la ciencia. ${ }^{13}$ Este principio se basaba en el supuesto de que "en la naturaleza todo tiene un telós, un fin completado $\mathrm{y}$ perfecto [...] [que] mantiene en una uniformidad de estructura y función a individuos distantes en el espacio y lejanos en el tiempo"; y que "parecía dar acceso

\footnotetext{
${ }^{11}$ Véase Marradi, A., “Esperimento, associazione, insieme non standard?", en Bettin, Giovanni (comp.), Politica e Società, Padua, Cedam, 1997.

${ }^{12}$ Pearson, K., op. cit., pp. 173-174.

${ }^{13}$ Dewey, J., op cit., pp. 51-52.
} 
nada menos que a la naturaleza de la realidad misma". Esta concepción clásica llevaba implícita la idea de propósito: "un principio regulativo finalístico que no es visible a los sentidos y debe tratarse de una fuerza ideal o racional [...] una fuerza causal espiritual, que por espiritual escapa a la percepción, pero que es aprehendida por una razón esclarecida."14

Al igual que en el caso de Dewey, el pensamiento de la naciente comunidad de estadísticos matemáticos, en particular el de Pearson —único que logró articularlo en una obra epistemológica de relevancia - refleja en muchos sentidos esta corriente antimetafísica fuertemente contestataria con respecto a las ideas tradicionales de "naturaleza”, “cosa en sí”, y “causalidad”.

El libro The Grammar of Science (1892), para muchos el más importante sumario epistemológico escrito en el período, es un verdadero elogio de la nueva visión de la ciencia y de la sociedad. ${ }^{15}$ En el prefacio a la primera edición, escrito en enero de 1892, Pearson afirma que la ciencia debía "llevar adelante una difícil guerra contra la metafísica y el dogma". Pero para cumplir este cometido era necesario "examinar atentamente sus fundamentos", sin esconder los propios defectos. ${ }^{16} \mathrm{~A}$ su entender, el lenguaje de la ciencia dominante — la física—, usado también en otras disciplinas, necesitaba un re-examen. Los textos elementales de ciencia no tenían valor educativo; no promovían la claridad lógica ni el ejercicio del "método científico". El físico, modelo que todos buscaban imitar, se encontraba a su juicio entrampado en las redes de pseudo-ciencias tales como la teología natural y el espiritualismo.

En la Inglaterra tardo victoriana - hacia fines del siglo XIX - Pearson devino la principal voz de la ciencia positivista. ${ }^{17} \mathrm{Su}$ discurso epistemológico, en el que la tradición empirista británica se amalgama con aspectos del poskantismo alemán y con ciertos elementos del racionalismo matematizante de Descartes, intentaba eliminar la

\footnotetext{
${ }^{14}$ Dewey, J., op cit., pp. 54-55.

${ }^{15}$ Véase Levine, G., "Science and Citizenship: Karl Pearson and the Ethics of Epistemology", en Modernism/ Modernity 3.3, 1996, pp. 137-143.

${ }_{16}$ Pearson, K., op. cit., p. xvii.

17 Levine, G., "Two Ways Not To Be a Solipsist: Art and Science, Pater and Pearson", in Victorian Studies 43, 1, 2000, pp. 7-41.
} 
confusión que imputaba tanto al pensamiento metafísico como a gran parte del pensamiento científico de su tiempo.

En este sentido, Pearson presenta su Grammar of Science como una obra destinada a estimular el libre pensamiento y orientada a exaltar el valor social de la ciencia; una fuente en la que los jóvenes estudiantes pudiesen encontrar una perspectiva libre de metafísica. Sin embargo, sus ideas eran entonces bastante radicales, y a pesar de haberse esforzado por presentarlas de modo "digerible" para sus contemporáneos, tal como él mismo reconoce cuando agradece a todos aquellos que lo habían ayudado a moderar su fervor retórico, fueron objeto de encendidas polémicas (recuérdese la típica tensión de la época —ya aludida en los primeros párrafos de este artículo - entre ciencia y teología natural). Intelectuales de la talla de Lord Balfour, influyente personaje de la cultura victoriana, reaccionaron críticamente contra las tesis expuestas en el libro, sosteniendo que la ciencia no tenía más autoridad en materia de conocimiento que las grandes tradiciones del pensamiento teológico. ${ }^{18}$ Su libro The Foundations of Belief (1894) representa una abierta crítica a la validez de los métodos científicos como medios para indagar y producir conocimiento en todas las áreas de la experiencia, así como un ataque frontal contra la cruzada de Galton, Pearson y otros en favor del darwinismo. Fue en este contexto que Dewey, como perspicaz observador, se ocupó de señalar que los "muchos esfuerzos sinceros y vitales" que se estaban produciendo para revisar las concepciones filosóficas tradicionales de acuerdo con las exigencias de la nueva ciencia se enfrentaban a un "recrudecimiento no menos perspicuo de las filosofías absolutistas." 19

Por otra parte, resulta interesante recordar que la batalla de Galton, Pearson y otros intelectuales anglosajones por establecer la autoridad de la ciencia en una cultura todavía predominantemente teológica, no descansaba únicamente en sus ventajas en términos de conocimiento y control sobre la naturaleza; se apoyaba principalmente en una fe iluminista en sus cualidades como vía para el mejoramiento de la sociedad. Pearson, por ejemplo, sostenía que la capacidad de aumentar la

\footnotetext{
${ }^{18}$ Levine, G., op. cit.

${ }^{19}$ Dewey, J., op. cit., pp. 59-60.
} 
felicidad social, promover el bienestar de la sociedad humana y reforzar su estabilidad era la única justificación aceptable de cualquier institución social o forma de actividad humana, incluyendo a la ciencia. En este sentido, declaraba que promover la difusión del conocimiento científico era fundamental para formar ciudadanos más responsables y, en consecuencia, lograr una sociedad más estable ${ }^{20}$ :

\footnotetext{
La ciencia moderna, en cuanto entrenamiento de la mente para el exacto análisis de los hechos, es un tipo de educación especialmente apta para la promoción de una ciudadanía sana ${ }^{21}$
}

Aunque con importantes discrepancias en cuanto a qué debía entenderse por "mejoramiento de la sociedad",22, también Dewey creía que "la nueva lógica [introducía] responsabilidad dentro de la vida intelectual"23, y de este modo contribuía a una ciudadanía más plena. El contenido ético de su filosofía, así como su preocupación por la política y por la educación, son cuestiones bien conocidas, y si bien con grandes diferencias de matices, constituyen aspectos comunes del tipo de "defensa de la ciencia" que tanto él como los estadísticos positivitas ingleses propugnaban. En este sentido, todo ellos compartían los puntos fundamentales de una lectura de la obra de Darwin y de sus consecuencias para su tiempo: "sin lugar a dudas, en el pensamiento contemporáneo el más poderoso disolvente de preguntas viejas, el principal catalizador de nuevos métodos, nuevas intenciones, nuevos problemas, es el que proviene de la revolución científica que alcanzó su clímax en $E l$ origen de las especies." 24

\footnotetext{
${ }^{20}$ Esta era una preocupación central de Pearson, que denunciaba "el peligro de que se retardase la difusión del conocimiento científico entre las personas no iluminadas, adulando el oscurantismo a través del descrédito del método científico" (Pearson, K., op. cit., p. 18). ${ }^{21}$ Pearson, K., op. cit, p. 9.

${ }^{22}$ Recuérdense a propósito del mejoramiento de la sociedad los compromisos eugenésicos de Galton, Pearson y otros estadísticos ingleses de la época.

${ }^{23}$ Dewey, J., op. cit., p. 59.

${ }^{24}$ Dewey, J., op. cit., p. 60.
} 


\section{Bibliografía}

Balfour, Arthur, The Foundations of Belief, Londres, Longmans y Green, 1894.

Darwin, Charles, On the Origin of Species, Londres, John Murray, 1859.

Dewey, John, "The influence of Darwinism on Philosophy", en The influence of Darwinism on Philosophy and other essays in contemporary thought, Nueva York, Henry Holt and Co., 1910 .

Fayter, Paul, "Strange New Worlds of Space and Time: Late Victorian Science and Science Fiction", en Lightman, Bernard (ed.), Victorian Science in Context, Chicago, University Press, 1997, pp. 256-280.

Galton, Francis, Natural inheritance, Londres, Macmillan, 1889.

Kjaergaard, Peter C., Defending Science: 'Genuine Scientific Men' and the Limits of Natural Knowledge. Aarhus, University Press, 2000.

Levine, George, "Science and Citizenship: Karl Pearson and the Ethics of Epistemology", en Modernism/Modernity 3.3, 1996, pp. 137-143.

Levine, George, “Two Ways Not To Be a Solipsist: Art and Science, Pater and Pearson", in Victorian Studies 43, 1, 2000, pp. 7-41.

Lightman, Bernard, "'The Voices of Nature': Popularizing Victorian Science", en Lightman, Bernard (ed.), Victorian Science in Context, Chicago, University Press, 1997, pp. 187-211.

Mackenzie, Donald A., Statistics in Britain, 1865-1930. The Social Construction of Scientific Knowledge, Edimburgo, University Press, 1981.

Malthus, Thomas R, Essays on the Principles of Population, Londres, J. Johnson, 1798.

Marradi, Alberto, “Esperimento, associazione, insieme non standard?", en Bettin, Giovanni (comp.), Politica e Società, Padua, Cedam, 1997.

Norton, B. J. , "Karl Pearson and Statistics: The Social Origins of Scientific Innovation”, Social Studies of Science 8, 1, 1978.

Pagano, Robert, Understanding statistics in the behavioral sciences, Pacific Grove, Brooks/Cole, 1998.

Pearson, Egon, Karl Pearson: An Appreciation of Some Aspects of his Life and Work, Cambridge, University Press, 1938. 
Pearson, Karl, The Grammar of Science, Londres, Scout, 1892.

Winter, Alison, "The Construction of Orthodoxies and Heterodoxies in the Early Victorian Life Sciences", en Lightman, Bernard (ed.), Victorian Science in Context, Chicago, University Press, 1997, pp. 24-50. 